\title{
Tracking Darkports for Network Defense
}

\author{
David Whyte Paul C. van Oorschot Evangelos Kranakis \\ School of Computer Science \\ Carleton University, Ottawa, Canada \\ \{dlwhyte, paulv, kranakis\}@scs.carleton.ca
}

\begin{abstract}
We exploit for defensive purposes the concept of darkports - the unused ports on active systems. We are particularly interested in such ports which transition to become active (i.e. become trans-darkports). Darkports are identified by passively observing and characterizing the connectivity behavior of internal hosts in a network as they respond to both legitimate connection attempts and scanning attempts. Darkports can be used to detect sophisticated scanning activity, enable fine-grained automated defense against automated malware attacks, and detect real-time changes in a network that may indicate a successful compromise. We show, in a direct comparison with Snort, that darkports offer a better scanning detection capability with fewer false positives and negatives. Our results also show that the network awareness gained by the use of darkports enables active response options to be safely focused exclusively on those systems that directly threaten the network.
\end{abstract}

\section{Introduction}

The Internet is saturated with "nonproductive" network traffic that includes an estimated 25 billion global intrusion attempts per day [17, 33]. A precursor to most of these intrusion attempts involves some form of reconnaissance activity to identify vulnerable systems or to determine the best point of access into a target network. Automated tools methodically probe large blocks of Internet address space seeking vulnerable systems for recruitment into botnets [8, 19, 5, 2]. Large numbers of worm-infected systems randomly scan the Internet searching for susceptible systems to exploit. Perhaps most worrisome for a network operator is when a determined adversary directs specific scanning activity solely against their network searching for weaknesses to provide them with an entry vector. This type of reconnaissance is typically precise, deliberate, and focused.

A variety of complex heuristics have been successfully developed to detect scanning activity including the observation of connection failures [11,23], statistical measures $[12,25]$, abnormal network behaviors $[28,31,6]$, and con- nections to network darkspace [7, 14]. Current scanning detection algorithms focus largely on observing and classifying external network behavior (i.e. incoming network connection attempts) to detect scanning systems, although many types of sophisticated scanning techniques (e.g. botnet scanning, slow scanning) make it difficult or impossible to accurately determine root-cause origins of scanning activity.

In contrast, exposure maps [29] were proposed to detect scanning activity by passively observing legitimate traffic and attack scans (active scanning) directed at a target, and especially observing how internal hosts respond to external connection attempts. Preliminary investigation suggested they were suitable for detecting sophisticated scanning activity directed at an enterprise network, with greater interest in what an adversary is searching for, than in who is scanning the network. Successful connections to internal systems would be characterized in exposure maps to define the currently active external interface to the network. In contrast to remote network security auditing techniques (e.g. Nmap [9]), exposure maps were asserted to facilitate an efficient, low-effort method to identify network vulnerabilities, with exposure status continually updated.

In this paper, we pursue these ideas and introduce darkports which we define as unused ports (i.e. ports with no service responding) located on active hosts. Darkports provide a method to detect in real-time unauthorized service offerings from a host; these may indicate a successful compromise (e.g. a darkport suddenly starts to respond to a connection request).

First, we validate a preliminary assertion from the position paper [29] that exposure maps are very effective at detecting both simple and sophisticated TCP scanning activity directed at an enterprise network. In a direct comparison, the exposure map scanning detection capability was significantly better than the well-known Snort [22] (i.e. lower false negative and positive rates and the ability detect additional sophisticated scanning activity).

Secondly, we show that the identification of darkports during the construction of the exposure maps provides network-centric knowledge enabling fine-grained automated responses, e.g. to identify and deny specific systems network access when they are found to be performing scanning activ- 
ity and thereafter trying to access a legitimate service in the network (common behavior for autorooters and worms [18]). This introduces the ability of selective automated response: a focused real-time active response option that limits the introduction of new access control rules to deny those scanning systems directly threatening network assets (i.e. those targeting actual services offered by the network). We emphasize the subtle point, that systems that scan for services not offered by the network are simply identified (i.e. scan recorded) but otherwise ignored (e.g. no access control rule introduced to block the associated source IP address). This ability to initiate selective automated response reduces network configuration changes, complexity errors (e.g. by avoiding a dramatic increase in router/firewall rules, and possibly leading to a self-imposed denial of service), and avoids unnecessary performance degradation of network security devices [4, 32].

Exposure maps and darkports differ from current scanning detection techniques as they rely on identifying the services offered by the network instead of tracking external connection events. The result is a scanning detection technique in which the utilized system detection state does not grow in proportion to the amount and fluctuation of external network traffic, but rather increases only with the number of services offered by the network, regardless of the size of the network and the external network activity. As an added benefit, maintaining information about internal hosts in the network instead of external host activity provides the necessary network-awareness to answer in real-time questions that should be asked after a scan is detected, such as "What information has been revealed as a result of the scan?", and "Has the network behavior changed?"

The remainder of this paper is organized as follows. Section 2 refines the basic idea of exposure maps and darkports. Section 3 discusses how exposure maps can be used for scanning detection and automated response. Section 4 describes our implementation, the evaluation dataset, and methodology. Section 5 presents our evaluation results, including a comparison to Snort, and discussion of advanced scanning heuristics. Section 6 discusses the scalability and stability of exposure maps, including resilience to attacks. Section 7 presents limitations of the technique. Section 8 reviews related work. We conclude in Section 9.

\section{Exposure Maps and Darkports}

We first describe the constituent components of exposure maps, how exposure maps are constructed and how they define the darkports within the network. We focus on exposure maps relative to TCP ports.

COMPONENT DESCRIPTION. Exposure maps passively identify the services which have been confirmed (using an observed response during a training period) as being offered by the hosts of a given network. TCP packets with the SYN flag set start the three-way connection handshake. When a connection request is sent to a specific destination IP address/port, if a service is bound to that port and the port is listening (open), the target host response is a packet with SYN ACK flag set, to start a session. Listening services, because they respond to connection attempts or incoming packets, leak information to scanners; they typically correspond to the active ports in a network and can be tracked in terms of what we define below as the HEM and the NEM. Once verified as permitted activity, the HEMs and NEM define the authorized access to individual hosts and the network.

More specifically, a host exposure map (HEM), associated with a fixed IP address (host), is the set of ports observed responding to external connection attempts within a predefined period. For each active host $i$ in the network, $H E M_{i}$ is a set of elements each of which begins with the IP address of $i$, followed by a port number $j$; there is such an element for each port $_{j}$ that has responded to a connection attempt within a predefined period. In symbols, we can abbreviate this as $H E M_{i}=\left\{I P_{i}\right.$ : port $_{j} \mid$ port $_{j}$ was observed responding $\}$.

The HEM is the externally visible interface of a host and can be considered to represent information leakage from the host that may reveal characteristics that can be used to exploit it. Subsequent to the training period, as additional ports respond to external connection attempts, by definition the HEM is augmented by these ports.

The network exposure map (NEM) is defined as the collection of HEMs in a given network $N$ at any given point in time. The NEM defines how we expect the network to respond to external connection attempts. In symbols, $N E M_{N}=\bigcup_{i \in N} H E M_{i}$. We will often drop the subscript $N$ in $N E M_{N}$ when the target network is implied by context. This also allows the natural definition of $N E M_{S}$ for any subnetwork $S \subset N$, i.e. where $S$ is a subset of the populated IP addresses in $N$. In an implementation, once the NEM has been built, the individual HEMs that comprise it can be checked for compliance with the network security policy. A NEM that complies with the network security policy is called a vetted NEM. We assume that any service (IP address/port pair) not compliant with the network security policy will, once detected, either be shutdown, or implicitly becomes part of the security policy. Thus, movement from a NEM to a vetted NEM is always possible.

We define the darkports on a given (real) host as those ports that have not been observed offering any services, and thus are not expected to accept external connection requests. ${ }^{1}$ The set of darkports for a host is the complement of its HEM. The set of darkports for a network is the union of the darkports on all its populated hosts. For example, a host with a HEM of only three TCP ports 22, 80, and 443 would have $2^{16}-3$ TCP darkports i.e., all TCP ports excluding these three. If a darkport responds to an external connection attempt, it becomes a trans-darkport. This occurs either when

\footnotetext{
${ }^{1}$ Although a connection attempt to any port at a darkspace IP address (no hosts assigned) will not accept a connection attempt, we restrict the term darkport to unused ports on a populated host address.
} 
a host offers a new service (whether authorized or rogue), or a connection is made to a service that was not accessed during the training period. Either event causes the HEM to expand, and by definition the NEM expands and will differ from the vetted NEM. Once a trans-darkport is detected, this change can be checked against the network security policy so that the vetted NEM can be updated or any unauthorized service can be stopped.

Exposure MaP Construction AND MainteNANCE. In summary, exposure maps are created by passively observing a target network's responses to incoming connection attempts (both legitimate connections and scanning attempts) over a training period. Every time a host responds to an external TCP connection attempt, the IP address and port of the host offering the service is recorded. During the training period, each host in the network will reveal services that it offers; the corresponding ports are recorded in its HEM. After the training period, the vetted NEM can be used to identify all the active hosts on the network by their representative HEMs. Thereafter during ordinary network operation, passive observation of network packets continues, and for each connection attempt (i.e. each TCP SYN packet) compliance with the vetted NEM is tested in real-time. If the services offered by a host expand beyond the vetted NEM, an alert is generated to provide notification that trans-darkports have been detected; this indicates that either the vetted NEM needs to be updated, or some form of unauthorized activity is occurring.

In general, an important consideration for any technique that requires a training period is that any existing malicious activity (e.g. unauthorized services) may become part of the baseline. In our particular case, a HEM can be verified against an existing network security policy to detect any unauthorized service offerings by the host. The required length of the training period will vary with each network environment depending on a number of factors including number of active hosts, network security policy, permitted user applications, and frequency of service usage; see Section 6 for further discussion.

\section{Applications of Exposure Maps and Dark- ports}

Exposure maps provide network-centric knowledge to enable a variety of security applications including scanning detection and automated response which are discussed in the subsections below. ${ }^{2}$

\subsection{Scanning Detection Using Exposure Maps}

Motivation. Panjwani et al. estimate that $50 \%$ of attacks against systems are preceeded by some form of net-

\footnotetext{
${ }^{2} \mathrm{~A}$ third application for exposure maps, not presented in this paper due to page limitations, is network discovery/asset classification and is discussed in [30].
}

work scanning activity [18]. Current scanning detection algorithms are generally designed to identify and classify suspicious network activity as scanning activity using attribution to a particular source or sources. These algorithms are effective at detecting wide-range reconnaissance activities that can be defined as the rapid scanning of large blocks of Internet addresses in the search for a specific service or vulnerability. This is characteristic of autorooters [26] and worm propagation. Autorooters are composite tools that augment basic port scanning functionality by launching an attack as soon as an open port is located on a target system [1]; they are often used for the rapid enrollment of vulnerable systems into botnets of tens or hundreds of thousands of compromised systems [2]. Simple scanning worms propagate by indiscriminately probing the Internet as rapidly as possible to locate and infect vulnerable systems. Scans from autorooters and scanning worms can usually be attributed to the true source as the scans themselves are the first stage of the actual exploit attempt (e.g. a response, from the target, to a TCP SYN connection request will start the exploit in the same session).

In contrast to such indiscriminate scanning, skilled adversaries will go to considerable lengths to mask their activities. Numerous sophisticated scanning techniques allow stealthy, focused scanning of a predetermined target (host and/or network); some of these make attribution to the scanning source impractical, rendering most current scanning detection techniques ineffective. The following techniques belong to this category.

Slow scanning activity against a network or host can be spread out over days or weeks. Over time, these scans will simply be lost in the network noise, never exceeding scanning detection thresholds (i.e. being outside of the allocated detection system state).

Indirect scanning occurs when an attacker uses one system (or systems) to scan a target and another system to attack the target. This separation defeats attribution attempts. If the scanning activity from the scanning system is detected (e.g. blocked at a network router, or by system administrator intervention), the attacker simply uses another scanning system. A slightly more sophisticated variation uses throw-away scanning systems, i.e. previously compromised systems that have little value to an attacker other than being able to provide a disposable platform. Any scanning activity traced back to the source, will be attributed to the owner of the compromised system.

Distributed scanning occurs when multiple systems act in unison using a divide and conquer strategy to scan a network or host of interest. Typically, one system will act as a central node and collect the scanning results from all participating systems. Distributing the scanning activity reduces the scanning footprint from any single system and thus reduces the likelihood of detection. An extreme version of distributed scanning involves an attacker using a botnet to scan a target in a coordinated manner resulting in very stealthy scans. A 
relatively small botnet of a few thousand systems can be used to scan thousands of ports or hosts with only a single packet sent from each bot.

Use of Exposure MAPS FOR ScANNING DetecTION. A vetted NEM is constructed as previously described. A connection attempt to any port-IP combination not present in the vetted NEM (i.e. a darkport or darkspace) is defined as an atomic scan event. The 5-tuple (source IP, destination IP, destination port, protocol, ${ }^{3}$ timestamp) of any atomic scan events is recorded for further analysis to secondary storage (hard disk) in the scanning activity log file. This approach requires only that the NEM information be maintained in system detection state (not the darkports or external connection requests), thus allowing detection of even very slow or distributed scans, using only a small amount of main memory (see Section 6). In contrast to most scanning detection techniques that rely on the identification and correlation of external connection events to detect scans, we thus do not require strategies like reducing the detection time window in which connection events are tracked or timeouts, to accommodate network traffic fluctuations. Each atomic scan event can result in one of three possible outcomes: (1) a probe directed against a darkspace address, (2) a probe against a darkport (note: such a host has a HEM), or (3) a probe sent to a host on an active port (an entry in the NEM).

Unlike most attribution-based scanning detection techniques, the scanning detection approach does not rely on identification of the scanning source to detect scans against a network. Thus, it can detect certain classes of sophisticated scanning techniques that make determining the root cause of the scanning activity impractical. However, this approach does not preclude us from the use of some form of attribution post scan detection. Scanning worm propagation and autorooters are two prevalent examples of scanning activities where immediately denying the scanning source access to the network is both relevant and important. In these cases, a successful scan (i.e. one triggering a response from a host) typically leads to an immediate attack from the scanning systems (see Section 5.2). Other post scan detection activities may include the use of heuristics to classify atomic scan events into their respective scanning campaigns. An example of such a heuristic is given in Section 5.1.2 to identify and correlate the atomic scan events that comprise a distributed scan.

\subsection{Automated Response using Exposure Maps}

Exposure maps can be used in an automated response application as follows. When a new connection request is observed, the destination IP address and port are compared with the vetted NEM to determine if there is a match (see Figure 1). If there is no match to an entry in the NEM, the connection is considered a scan and the source IP address is added as an element in a scanners list (implemented e.g., using a

\footnotetext{
${ }^{3}$ Here, the protocol is TCP.
}

hash table). The 5-tuple (as in Section 3.1) that characterizes the connection attempt is then recorded as an atomic scan event in the scanning activity log file.

On the other hand if there is a match, the source IP is checked against the scanners list. If the source IP address matches an entry in the list, the 5-tuple that characterizes this connection attempt is recorded and connection should be dropped as this entity has previously undertaken reconnaissance activity against the network. Our implementation is passive and only produces alerts that could enable some form of containment (e.g. ACL change), but does not actually do the latter; one option would be to integrate this application on a network device capable of performing containment such as a firewall. If the source IP address does not match an entry in the scanners list, the connection is permitted; the entity has no previous history of scanning activity and is connecting to a valid offered service.

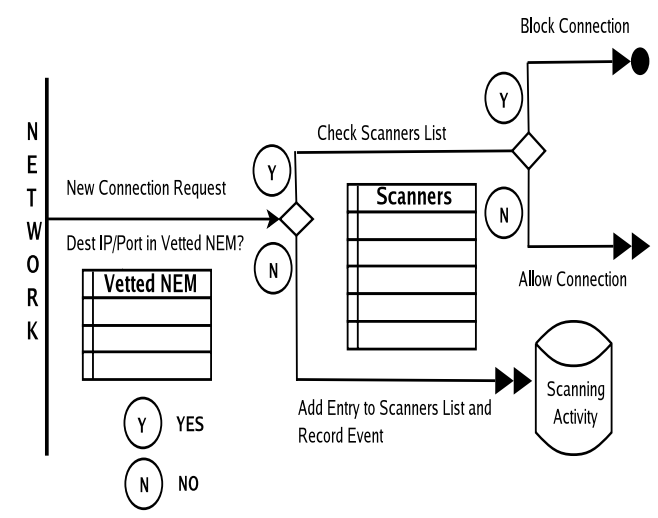

\section{Figure 1. Exposure Map Automated Response Logic.}

The vetted NEM provides context to determine if an incoming connection request is part of a scanning campaign and whether it will likely elicit a response. This information provides us with the precision to limit containment to (e.g. automatically block) only those scanning systems targeting services offered by the network (see Section 5.2). Containment could alternately be performed using a number of network devices including firewalls, routers, or intrusion prevention systems using current scanning detection techniques. However, given the prevalence of scanning activity, frequent dynamic updates to these core network devices would be required in order to stop attacks in real-time, and would pose a number of challenges. For instance, Bobyshev et al. [4] have shown that the size of access control lists (ACLs) and the frequency of dynamic updates can significantly impact router CPU utilization and forwarding capabilities. Furthermore, the addition of multiple blocking rules may make ACLs and configuration files cumbersome and hard to vet by network personnel. In fact, frequent configuration changes to these network devices may actually decrease the overall security posture of the network over time [32]. Our technique 
allows a precise active response option to be taken exclusively against the most critical known threats to the network namely, those scanning systems targeting services offered by the network. Scanning systems trying to access services not offered by the network are noted (i.e. in the scanning activity $\log$ and the scanners list) but no action is needed or taken to block the connection.

Our analysis on a four-week network data set reveals a majority of scanning attempts directed against services not offered by the network. In the instances when the scanning was directed against a service offered by the network, an attack usually followed (see Table 4 and discussion in Section 5.2). Thus, our approach can significantly reduce the frequency and number of updates to the ACLs of network security devices while providing a measured and robust security response to real-time threats.

\section{Evaluation: Dataset and Methodology}

To evaluate how darkport observation can be applied, we developed and tested a software implementation. The software is installed on a commodity PC connected to the network by a 10/100 network interface card.

CCSL DATASET. The CCSL network is a small university research network of 62 Internet reachable addresses connected to a university Internet accessible Class B network. All systems access the Internet through a firewall not permitting inbound connections unless initiated by an internal system. The CCSL dataset consists of four weeks (September 2006) of network traffic collected in pcap files in front of the network firewall.

Evaluation Methodology. We tested scanning detection and selected automated response capabilities on the CCSL network dataset; its network boundaries are known, allowing the NEM to be validated against a known network security policy. Additionally, having access to the full network traces, post scan detection analysis was possible to confirm our experimental results when comparing actual scanning detection capability with Snort.

\section{Evaluation Results}

We tested the ability of exposure maps to perform scanning detection by performing a side-by-side comparison with Snort [22]. We then show how exposure maps can be used to detect sophisticated scanning activity and analyze the effectiveness of using the exposure map scanning detection capabilities to perform a real-time fine-grained automatic response to attacks.

\subsection{Results: Scanning Detection}

As discussed, the CCSL network dataset has a NEM comprised of three HEMs (see Table 1). Two of these have three active ports; the third has one active port. The NEM thus has in total seven port/IP entries.

\subsubsection{Scanning Detection Comparison with Snort}

We compared scanning detection results with Snort on the CCSL network dataset. We used a one-day training period to construct the NEM; it stabilized within the first 20 hours of network traffic. Snort's preprocessor, sfPortscan [21], performs port scanning detection and allows operations on decoded packets before they are sent on to the Snort detection engine. sfPortscan provides the capability to detect TCP, UDP, and ICMP scanning; its sensitivity is set using the sense level parameter (low, medium, or high). We focused on TCP scans at sense level high. Three types of scans were detected by Snort in the CCSL dataset: 1) portscans (single host scans multiple ports on a single host); 2) distributed portscans (multiple hosts scan multiple ports on a single host); and 3) portsweeps (single host scans a single port on multiple hosts).

Table 1. Details about NEM for CCSL network.
\begin{tabular}{|r|r|r|}
\hline Host & TCP Ports & Description \\
\hline \hline 10.0 .0 .1 & $25,631,993$ & SMTP/IPP/IMAP \\
\hline 10.0 .0 .2 & $22,80,443$ & SSH/HTTP/SSL \\
\hline 10.0 .0 .3 & 22 & SSH \\
\hline
\end{tabular}

The implementation detected 740885 atomic TCP connection events (scans). The upper bound on the possible TCP scanning footprint is $E=62 * 2^{16}$. The actual scanning footprint we detected was $A=2342$ unique TCP port/IP combinations (including all seven entries in the NEM). With 26 live systems in the network, the number of darkports is $D P=26 * 2^{16}-7$ (the seven entries in the NEM are excluded). To compare exposure maps with Snort, we applied Snort's scan definitions to group the scans ${ }^{4}$ we detected.

Snort detects scans by counting RST packets from each perceived target during a predetermined timeout interval [13]. Before declaring a scan, 5 events (i.e. RST packets) are required from a given target within a window. The sliding timeout window varies from 60 to 600 seconds by sensitivity level; at the highest level, an alert will be generated if the 5 events are observed within 600 seconds. Exposure maps do not employ a timeout window; the 5-tuple of atomic scan events are simply recorded and stored, whereafter a number of heuristics can be used to classify the scans detected (see Section 5.1.2). On the other hand, Snort does not require a training period.

Table 2 summarizes the results. Snort detected a total of 8052 scans initiated by 322 unique scanning systems, while the NEM detected 8513 scans initiated by 813 unique scanning systems - all of the 8052 scans detected by Snort, and an additional 461 scans initiated by 461 unique systems not identified by Snort. These are denoted other scans in Table 2; they encompass a variety of scanning techniques not

\footnotetext{
${ }^{4}$ Recall that a scan is defined by the NEM as an atomic TCP connection attempt.
} 
Table 2. Scanning Detection Comparison. " $+n$ " are scans that are false positives.

\begin{tabular}{|l|r|r|}
\hline & Snort & $\begin{array}{r}\text { Exposure } \\
\text { Maps }\end{array}$ \\
\hline \hline Port Scans & $127+1$ & 127 \\
\hline Distributed Port Scans & $54+14$ & 54 \\
\hline PortSweeps & $7871+42$ & 7871 \\
\hline Other Scans & 0 & 461 \\
\hline False Positives (total) & 57 & 0 \\
\hline False Negatives & 461 & 0 \\
\hline Unique Scanners & 322 & 813 \\
\hline
\end{tabular}

included in the sfPortscan scanning definitions, e.g., scans from a single host to a single port on a single host, slow scans with scan intervals of greater than 15 minutes, and a single host scanning multiple ports on multiple hosts. In the next section, we discuss in detail some heuristics used to detect distributed scans. 57 of the scans Snort detected were false positives, the majority caused by legitimate RST packets traversing the network. At the high sense level, a moderate amount of false positives are expected by normal network activity.

False Negatives. We relied on the output of Snort to provide a baseline of the scanning activity within the dataset. As mentioned, we detected all scans identified by Snort, plus another 461 scans which Snort missed. Thus relative to Snort, for this dataset, our analysis for exposure maps (Table 2) revealed no false negatives.

Exposure maps (once vetted against the security policy) define the authorized access to the network from external sources. Connections attempts or scans outside these maps are considered a possible scans. Scans directed against a port/IP combination contained in the NEM are not considered a scan but rather a connection attempt to a valid service; this might potentially then be a source of false negatives, and to claim otherwise (i.e. zero false negatives in general) would imply unknowable knowledge of the intent of the party requesting the connection. For instance, a scan to port 443 of host 10.0.0.2 (see Table 1) in the CCSL network would not be recorded as a scan. In practice, although this specific event in a scanning campaign would not be detected, the overall scanning campaign would likely be detected using exposure maps for scan detection, as in most cases we would expect with high probability scans to occur against other hosts in the network not offering SSL (i.e. port 443 darkports). Scanning activity directed solely at the HTTP server would remain undetected and be a source of false negatives. However, we expect that would be an actual attack rather than scanning activity; we do not claim that exposure maps can detect attacks (that are not preceded by scans).

FALSE Positives. Through user error or misconfigura- tion, a connection attempt might be made to a host or service not offered by the network. In this instance, the intent of the connection attempt was not to scan some portion of the network, but rather it is simply a failed attempt to access a legitimate service. Regardless, this activity would be classified as a scan as an attempt was made to connect to a host/port pair not listed in the NEM. Again, given that there is no way to measure the intent of a connection attempt, we must classify these events as scans. While no false positives occurred in our CCSL dataset test (vs. 57 by Snort), we do not claim this in general. False positives will be generated whenever new legitimate services are introduced on the network or services are utilized which were not accessed during the training period (with identification as a trans-darkport until the service has been added to the vetted NEM). We expect trans-darkports to occur infrequently in tightly controlled enterprise environments (e.g. in most government departments, financial, and health care).

\subsubsection{Exposure Map Advanced Scanning Detection}

The scanning detection application identifies connection attempts to darkports within a network, with a 5-tuple extracted from each atomic scan event and recorded in a log file, from which a number of heuristics can be developed to help classify and correlate these events into respective scanning campaigns. Here, we give a few examples of such heuristics to detect distributed scanning attempts.

Table 3. Three Detected Distributed Scans.
\begin{tabular}{|c|c|c|c|}
\hline $\begin{array}{c}\text { \# of } \\
\text { Scanners }\end{array}$ & $\begin{array}{c}\text { Scanned } \\
\text { Ports }\end{array}$ & $\begin{array}{c}\text { \# of Hosts } \\
\text { Scanned }\end{array}$ & $\begin{array}{c}\text { Follow-on } \\
\text { Attack }\end{array}$ \\
\hline \hline 3 & 80 & 62 & No \\
\hline 11 & 22 & 62 & Yes \\
\hline 9 & 25,53 & 62 & Yes \\
\hline
\end{tabular}

Attackers may disperse the scanning activity among several sources to reduce the overall scanning footprint in an effort to evade detection. To detect distributed scanning we propose classifying the scan events using the following criteria. 1) Scanning events and target destination ports. The number of scanning events per unique source IP address is determined, through analysis of the scanning log, over a configurable time interval (e.g. seconds, days). Similar amounts of scanning events from individual sources are grouped into clusters, which are then grouped by target destination ports. This final comparison reveals scanning systems that share the same scanning frequency (i.e. number of scan packets per unit time) and target service. We consider clusters of three or more scanning sources that target the same destination ports as a distributed scan; the number of systems in a cluster is configurable. 2) Source IP proximity and target destination ports. Scanning events are first sorted by unique source IP address. Scanning sources in the same one-quarter class $\mathrm{C}$ 
subnet address range (e.g. /26) are grouped into a cluster. These clusters are then grouped by target destination ports. This reveals scanning systems sharing a similar contiguous address space (which could indicate a single entity $0 w n s$ the scanning systems) and target (i.e. service). Again, we consider clusters of three or more scanning sources that target the same destination ports as a distributed scan.

Using these distributed scan heuristics, we detected three distributed scans in the CCSL network dataset (see Table 3). The first consisted of three source IPs targeting port 80 (HTTP). The scanning campaign was directed against the entire IP address range of the CCSL network (i.e. 62 systems). Once the scanning activity completed, no attacks were detected from these scanning sources. In fact, the only network activity exhibited by the systems participating in the distributed scanning campaign in the network trace was this specific distributed scan. The second distributed scan consisted of 11 systems targeting port 22 ( $\mathrm{SSH})$. The scan was also directed at the entire IP address range. Two of the hosts in the CCSL network offer services on port 22. In contrast to the first distributed scan, two of the scanning systems attacked both systems in the CCSL network that offered the service (c.f. Table 1). The third distributed scan detected consisted of 9 scanning systems targeting ports 53 (DNS) and 25 (SMTP). Two of the hosts in the network offer services on ports 25 and 53 respectively. Again, all hosts in the CCSL network were scanned with an attack immediately following on the system that offered port 25.

The distributed scanning detection heuristics described above illustrates how atomic scan events detected and recorded through exposure maps can be processed to detect sophisticated scanning activity. Other heuristics may be developed that use the raw output from exposure maps to identify other types of simple or sophisticated scanning activity (e.g. slow scanning).

\subsection{Results: Active Response}

Of the 813 scanners detected by the NEM in the CCSL dataset, 66 launched a total of 15301 scans intermingled with attacks (unsuccessful) against the network, e.g., repeated attempts to relay mail through the mail server, and attempted logins to an SSH service. Mail relaying is prohibited by our mail server and the responses from the mail server to the attacking system indicate that no relaying occurred; analysis of the network traces also showed that the repeated SSH login attempts were all unsuccessful. Some of these systems scanned and attacked multiple services; this explains why the number of scan/attack entities in Table 4 is 121 , while the actual number of unique IPs addresses was 66 . With the exception of a single distributed scan (see Table 3), two characteristics of this activity occurred: (1) scanning was always the precursor to the actual attack, and (2) whenever a scan was directed against a service offered by the network (i.e. entry in the NEM), an attack followed once a response to the scan was sent. This "scan then attack" activity fits the profile of autorooter or worm activity as previously described. The attacks were directed against four services offered by the network: SMTP, HTTP, SSL, and SSH.

Table 4. Scan Activity as Prelude to Attack.

\begin{tabular}{|l|c|r|}
\hline NEM Entry & $\begin{array}{c}\text { Scan/Attack } \\
\text { Entities }\end{array}$ & $\begin{array}{r}\text { Scans or } \\
\text { Attacks }\end{array}$ \\
\hline \hline $10.0 .0 .1: 25$ & 5 & 5 \\
\hline $10.0 .0 .1: 80$ & 12 & 18 \\
\hline $10.0 .0 .1: 443$ & 3 & 3 \\
\hline $10.0 .0 .2: 22$ & 40 & 4545 \\
\hline $10.0 .0 .2: 80$ & 17 & 120 \\
\hline $10.0 .0 .2: 443$ & 4 & 9 \\
\hline $10.0 .0 .3: 22$ & 40 & 10601 \\
\hline
\end{tabular}

Without the knowledge of what services are offered and in active use by the network, in a standard perimeter defense all 813 scanning system source IPs over the four week period might need to be blocked at the router or firewall. The NEM provides up-to-date knowledge of the external interface of the network, indicating which minimal set of scanning systems should be blocked. The NEM, coupled with the technique of Section 3.2, would require that only 66 source IP addresses be denied access. This represents a $92 \%$ reduction in the number of dynamic updates to the network security ACLs.

\section{Scalability and Stability of Exposure Maps}

The size of exposure maps will be determined by numerous factors, the two most important being the number of distinct hosts using the monitored link (or network), and the variety of applications those hosts use. The smaller the NEM, the less detection system state that needs to be maintained and the greater the scalability.

The resource consumption of exposure maps includes system detection state and disk storage. The former refers to storage for the features extracted from network events that must be maintained at all times in main memory, providing the wireline speed context to build and maintain the exposure maps as well as perform its various applications. ${ }^{5}$ A number of techniques are used by other network-based scanning detection approaches to limit their use of allocated system resources (CPU cycles, main memory, disk storage), e.g., connection timeouts, reduction of monitoring windows, fixed sized memory buffers and analyzing only certain events/protocols. The disk storage usage by exposure maps will depend on the detected scanning activity, increasing with the number of atomic scan events recorded. We now discuss the system detection state and disk storage requirements

\footnotetext{
${ }^{5}$ Depletion of this finite resource, due to traffic volume or an intentional DoS attack, can overload and defeat a detection system. Resilience to attack is discussed separately; see end of Section 6.
} 
for the various applications of exposure maps, and computational overhead.

SCANNing Detection. For scanning detection with exposure maps, (1) the NEM must be constructed and maintained, and (2) atomic scan events must be written to disk in the scanning activity $\log$ file. To understand the amount of detection system state for (1), consider the scenario of a network that has a NEM consisting of 10000 entries (e.g. 500 active systems with an average of two open services per system). Each NEM entry contains six bytes: four for IP address and 2 for port. The total memory footprint for this NEM is $6 * 10000 \approx 60 K$ bytes plus additional overhead for storage in a data structure (e.g. hash table, Bloom filter). Thus with an allocation of $100 \mathrm{~K}$ or $200 \mathrm{~K}$ in system detection state, we could perform scanning detection for this enterprise network. As new connection requests are received, a single lookup is performed on the NEM with the destination IP and port fields from the incoming request to determine if there is a match. The small amount of detection system state coupled with the minimal computational overhead required to determine if an incoming connection request matches a port/IP pair in the NEM (i.e. a single lookup) make this technique suitable for use at wire speed even in large enterprise environments.

To estimate the disk storage required for atomic scan events in the scanning event log file, we examined the results from the CCSL dataset. The unoptimized 5-tuple that represents each atomic scan event, in character delimited ASCII, requires 44 bytes of storage. In contrast, to store the 780885 atomic scan events detected in the CCSL dataset (4 week period) in the scanning activity log file would take 33Mbytes. Assuming the dataset represents an average level of scanning activity, an entire year of scanning activity for the CCSL network $(\approx 391$ Mbytes) could be stored on a single CD or USB key.

AUtOMATED Response. The automated response application is more expensive on system detection state than the scanning detection application due to the scanners list (recall Section 3.2). Each entry in the scanners list requires an additional 4 bytes (plus additional overhead for the hash table data structure). As connection requests are received, an additional lookup is required (i.e. a check against both the NEM and the scanners list) to determine if the source IP address matches an entry in the scanners list. We detected 831 scanners in the CCSL dataset. With an additional allocation of less than $4 \mathrm{~K}$ in system detection state, we could enable the automated response application. Over time, the scanners list will grow and would have to be managed (reduced) so that some predetermined limit of system detection state was not exceeded.

STABILITY. The stability of a NEM will vary greatly depending on the environment in which it is used. In an enterprise network with a tight network security policy (e.g. government, finance, health care), we would expect the NEM to stabilize quickly and thus be suitable for use in a scanning detection technique. As noted in Section 5.1.1, in our uni- versity lab network the NEM stabilized in 20 hours. In other environments, service usage may vary by day of the week. In a network environment with an open network security policy, the NEM may scale but perhaps stabilize more slowly as new hosts continually enter and leave the network (e.g. mobile users) or if new applications and services are continually being added to client systems (e.g. P2P file sharing, open proxies).

DOS ATTACKS. A potentially serious attack on many scanning detection mechanisms is one that specifically targets the detection system. In this context, we review the general construction and maintenance of basic exposure maps, plus the two main applications considered (scanning detection, and automated response).

The construction and maintenance of basic exposure maps appears resilient to DoS attack. Incoming scans (bursts or sustained activity) do not increase an exposure map's size (i.e. the number of HEM entries), which reflects only the number of services offered by the corresponding host. Incoming scans do need to be passively monitored, and connection requests are checked for matches against the NEM; however, the processing required for this is minimal, and we would expect any problems caused by volume of requests to cause other elements of a network to fail, e.g., having adverse affect on core network devices such as routers, or firewalls.

In the scanning detection application, secondary storage may be adversely affected by a large botnet DoS effort, because detected scanning activity is recorded. For example, for a 100,000 system botnet executing a scanning campaign on a target network, three simultaneous scans by each bot would consume 13.2Mbytes in the scanning activity log. A sustained scanning effort by such a botnet would exhaust disk storage in most networks. However, such an attack would also likely cause core network devices to fail as noted above.

The automated response application would experience the same impact on disk storage as the scanning detection application, plus system detection state would be consumed for source IP addresses added to the scanners list (as incoming connection requests to port/IP combinations outside the NEM result in new scanners list entries). A botnet of size 100,000 would consume 400Kbytes of (scanner list) system detection state; this state consumption does not increase after the first scan from each source IP address. The most successful attack would likely be an attacker intentionally trying to exhaust scanner list state by spoofing source IP addresses during a large scanning campaign; this could adversely affect the platform executing the automated response application.

\section{Limitations}

NON-STANDARD PORTS. One of the strengths of the exposure map approach (and all discussed applications herein) is that it need only maintain very little state when operational. It need not inspect or decode the content of a TCP connection, but only to observe external connection attempts (i.e. SYN packets) and record the IP address and source port if 
there is a response (SYN-ACK). Thus, exposure maps use port numbers to identify the offered service. Although port numbers are a good indication of the type of service offered, users may choose to install services that use non-standard ports, e.g., an HTTP server using port 8080 or 8000 instead of port 80 . Of course, use of non-standard ports may limit access as client systems must know the listening port number before accessing the service. In the case of creating a NEM for scanning detection, this issue is less of a concern; nonstandard port usage should be detected after training when the NEM is vetted.

\section{Related Work}

The basic idea of exposure maps was introduced in a position paper [29], and developed as an example of an attribution-free scanning detection technique. Preliminary analysis revealed that it could detect both sophisticated and simple forms of network scanning activity. Although not tested, exposure maps were also proposed to detect changes in the services offered in a network which a network operator could verify as either authorized activity or an indication of a successful attack.

Active scanning ${ }^{6}$ software, both open source and commercial, allows a security audit on a host or network $[9,27,20]$. Active scanning can be an integral part of a security audit to confirm that a host or network is in compliance with the network security policy. This activity however, can be costly in terms of human resources as it requires personnel to perform the required scanning activity (i.e. configure and operate the software) and interpret the results. Furthermore, active scanning provides only a snapshot in time of the active hosts and services in a network. Any new hosts or services offered by the network will only be detected at the next scheduled active scanning session.

Passive scanning techniques continuously monitor the hosts and services available in a network. Extrusion detection [3] refers to identification of unauthorized internal network activity by inspecting outbound network traffic; its ultimate goal is the identification of outgoing attack attempts from compromised internal systems in order to stop them from reaching their target. The passive asset detection system (PADS) is signature-based passive detection software with a rules engine to identify hosts and services running on a network by inspecting outbound network traffic [24]. It was created to provide supplementary information when performing active scanning of a network. Snort is an open source IDS that has scanning detection capability [22] through the use of the Snort preprocessor sfPortscan [21], by observing the RST packets within the network for a predetermined timeout window [13]. If five RST packets are detected from a suspected target within a configurable timeout window, an alert is generated.

\footnotetext{
${ }^{6}$ Active scanning involves injecting packets into the network in order to elicit some observable response.
}

Both Leckie et al. [12] and SPICE [25] use probabilistic models to detect scanning activity. These approaches attempt to assign connection probabilities to internal hosts based on the observation within normal network traffic conditions as a benchmark. Scanning systems are detected as they are assigned probabilities based on their current connection behavior which is measured and compared against the a priori connection probabilities assigned to the internal hosts. Jung et al. [11] use their Threshold Random Walk algorithm to identify scanning hosts, based on the observation that scanning systems will contact hosts and ports that are not available more often than benign systems.

Network darkspace is the unused IP addresses in a network and thus it should have no legitimate network activity directed to it; connection attempts to IP addresses that have no hosts assigned to them is considered anomalous. A number of commercial products (e.g. [14, 7]) make use of network darkspace to detect malicious network activity. A darknet is typically a large unused block of Internet-routable darkspace monitored for inbound packet activity. The larger the darknet, the better the darknet's ability to detect scans and attacks during an observation period [16, 15]. A related but subtly different approach by Harrop et al. [10] uses greynets, defined as regions of darknet address space that contain some active systems (i.e. some of the IP addresses in the darknet are assigned to active hosts). One of the motivations is that it is not possible for most enterprise network operators to have large regions of contiguous unused address space assigned to them. However, it would be useful to have some means to detect anomalous events if dark space was available on the network. Interspersing valid light (i.e. used) and dark (i.e. unused) addresses throughout a network will make it difficult for malware to avoid targeting greynet addresses and thus avoiding detection.

\section{Concluding Remarks}

We are the first to exploit the use of exposure maps and introduce the concept of darkports. In contrast to darknets and greynets $[10,7,14]$, even densely populated enterprise networks can make use of exposure maps as they exist on live hosts. The overall exposure map technique is based on a simple premise that is efficient to implement - it requires the passive observation, recording, and maintenance of a list of the services offered by the hosts in a network. This simplicity translates into a very efficient use of system detection state and computational resources that easily scales for use in large enterprise and backbone networks.

Exposure maps can be used to perform scanning detection and enable fine-grained automated responses to deny access only to those scanning systems that directly threaten hosts in the network. During our evaluation, our implementation of the exposure map scanning detection application had fewer false positives and negatives in a direct side-by-side comparison with Snort. The exposure map scanning detection approach, through the passive recording of all the services of- 
fered by the network, provides an awareness of active hosts, network darkspace, and darkports allowing network-centric context that increases the fidelity of scanning detection.

In an open network environment, the diversity of user population and permitted activity may make the enforcement of a single comprehensive network security policy impractical. Furthermore, mobile or transient users may make determining a stable baseline of all the services offered by hosts in the network infeasible. In such environments, exposure maps remain flexible enough to be configured to monitor a subset of the network to protect core network assets. A NEM could be composed of a single HEM (e.g. for host-based intrusion detection) or several HEMs (e.g. web server farm), allowing a network operator to focus on these mission critical servers. We have developed a full implementation of this approach in software that will be made available to the public.

\section{References}

[1] G. Bakos and V. Berk. Early detection of Internet worm activity by metering ICMP destination unreachable activity. In SPIE Conference on Sensors, and Command, Control, Communications and Intelligence, April 2002.

[2] P. Barford and V. Yegneswaran. An Inside Look at Botnets. Special Workshop on Malware Detection, Advances in Information Security, Springer Verlag, 2006.

[3] R. Bejtlich. Extrusion Detection, Security Monitoring for Internal Intrusions. Addison Wesley, first edition, 2006.

[4] A. Bobyshev, P. DeMar, and D. Lamore. Effect of Dynamic ACL (Access Control List) Loading on Performance of Cisco Routers. In Computing in High Energy Physics, Feb. 2006.

[5] D. Dagon, C. Zou, and W. Lee. Modeling botnet propagation using time zones. In Proceedings of the 13th Annual Network and Distributed System Security Symposium (NDSS'06), February 2006.

[6] D. Ellis, J. Aiken, K. Attwood, and S. Tenaglia. A behavioral approach to worm detection. In Proceedings of The Workshop on Rapid Malcode, 2003.

[7] Forescout Technologies Inc, Forescout product. http://www. forescout.com/wormscout.html.

[8] F. C. Freiling, T. Holz, and G. Wicherski. Botnet tracking: Exploring a root-cause methodology to prevent distributed denial-of-service attacks. In ESORICS, pages 319-335, 2005.

[9] Fyodor. Remote OS detection via TCP/IP stack fingerprinting. Phrack, 54, December 1998.

[10] W. Harrop and G. Armitage. Greynets: a definition and evaluation of sparsely populated darknets. In Proceedings of the 2005 ACM SIGCOMM Workshop on Mining Network Data, pages 171-172, 2005.

[11] J. Jung, V. Paxson, A. Berger, and H. Balakrishman. Fast portscan detection using sequential hypothesis testing. In IEEE Symposium on Security and Privacy, pages 211-225, 2004.

[12] C. Leckie and R. Kotagiri. A probabilistic approach to detecting network scans. In Eighth IEEE Network Operations and Management Symposium (NOMS 2002), pages 359-372, 2002.
[13] B. Malmedal. Using netflows for slow portscan detection. Master's thesis, Department of Computer Science and Media Technology, Gjovik University College, 2005.

[14] Mirage Networks. Mirage NAC. http://www.mirage networks.com.

[15] D. Moore. Network telescopes: Tracking denial-of-service attacks and Internet worms around the globe. In LISA, 2003.

[16] D. Moore, G. Voelker, and S. Savage. Inferring Internet denial of service activity. In 10th USENIX Security Symposium, 2001.

[17] R. Pang, V. Yegneswaran, P. Barford, V. Paxson, and L. Peterson. Characteristics of Internet background radiation. In The Internet Measurement Conference IMC, 2004.

[18] S. Panjwani, S. Tan, K. Jarrin, and M. Cukier. An Experimental Evaluation to Determine if Port Scans are Precursors to an Attack. In International Conference on Dependable Systems and Networks, July 2005.

[19] M. A. Rajab, J. Zarfoss, F. Monrose, and A. Terzis. A multifaceted approach to understanding the botnet phenomenon. In Internet Measurement Conference 2006 (IMC'06), Proceedings of, October 2006.

[20] RemoteScan Corporation, RemoteScan. http://www.remotescan.com

[21] D. Roelker, M. Norton, and J. Hewlett. sfPortscan. 2004. http:// cvs.snort.org/viewcvs.cgi/snort/doc/ README. sfportscan?rev $=1.6$.

[22] M. Roesch. Snort - lightweight intrusion detection for networks. In LISA, 1999.

[23] S. Schechter, J. Jung, and A. Berger. Fast detection of scanning worm infections. In 7th International Symposium on Recent Advances in Intrusion Detection (RAID 2004), September 2004.

[24] M. Shelton. Passive Asset Detection System. (PADS). http://passive.sourceforge.net.

[25] S. Staniford, J. Hoagland, and J. McAlerney. Practical automated detection of stealthy portscans. In 7th ACM Conference on Computer and Communications Security, 2000.

[26] M. Tanase. Introduction to Autorooters: Crackers Working Smarter, not Harder. SecurityFocus. http://www. securityfocus.com/infocus/1619.

[27] Tenable Network Security, Inc., Nessus Vulnerability Scanner. http://www.nessus.org.

[28] D. Whyte, E. Kranakis, and P. van Oorschot. DNS-based detection of scanning worms in an enterprise network. In Proc. of the 12th Annual Network and Distributed System Security Symposium, Feb. 2005.

[29] D. Whyte, P. van Oorschot, and E. Kranakis. Exposure Maps: Removing Reliance on Attribution during Scanning Detection. Position paper (5 pages). In USENIX HotSec 2006, Aug. 2006.

[30] D. Whyte, P. C. van Oorschot, and E. Kranakis. Tracking darkports for network defence. Technical report, School of Computer Science, Carleton University, TR-07-04, February 2007.

[31] M. Williamson. Throttling viruses: Restricting propagation to defeat malicious mobile code. In 18th Annual Computer Security Applications Conference (ACSAC), 2002.

[32] A. Wool. A Quantitative Study of Firewall Configuration Errors. IEEE Computer, 37(6):62-67, 2004.

[33] V. Yegneswaran, P. Barford, and J. Ullrich. Intrusions: Global characteristics and prevalence. In SIGMETRICS, 2003. 